\title{
Diverse Regimes of Mode Intensity Correlation in Nanofiber Random Lasers through Nanoparticle Doping
}

\author{
Martina Montinaro, ${ }^{\dagger}$ Vincenzo Resta, ${ }^{\dagger}$ Andrea Camposeo, ${ }^{*}$, Maria Moffa, $^{\ddagger}$ Giovanni Morello, ${ }^{\dagger}$
} Luana Persano, ${ }^{\ddagger}$ Karolis Kazlauskas, ${ }^{\S}$ Saulius Jursenas, ${ }^{\S}$ Ausra Tomkeviciene, " Juozas V. Grazulevicius," and Dario Pisignano*, $*, \perp_{(\mathbb{0}}$

${ }^{\dagger}$ Dipartimento di Matematica e Fisica "Ennio De Giorgi”, Università del Salento, via Arnesano, I-73100 Lecce, Italy

${ }^{\ddagger}$ NEST, Istituto Nanoscienze-CNR, Piazza San Silvestro 12, I-56127 Pisa, Italy

${ }^{\S}$ Institute of Applied Research, Vilnius University, Saulètekio 3, LT-10257 Vilnius, Lithuania

"Department of Polymer Chemistry and Technology, Kaunas University of Technology, Radvilenu Plentas 19, LT-50254 Kaunas, Lithuania

${ }^{\perp}$ Dipartimento di Fisica, Università di Pisa, Largo B. Pontecorvo 3, I-56127 Pisa, Italy

\section{Supporting Information}

\begin{abstract}
Random lasers are based on disordered materials with optical gain. These devices can exhibit either intensity or resonant feedback, relying on diffusive or interference behavior of light, respectively, which leads to either coupling or independent operation of lasing modes. We study for the first time these regimes in complex, solid-state nanostructured materials. The number of lasing modes and their intensity correlation features are found to be tailorable in random lasers made of light-emitting, electrospun polymer fibers upon nanoparticle doping. By material engineering, directional waveguiding along the length of fibers is found to be relevant to enhance mode correlation in both intensity feedback and resonant feedback random lasing. The here reported findings can be used to establish new design rules for tuning the emission of nanolasers and correlation properties by means of the compositional and morphological properties of complex nanostructured materials.
\end{abstract}

KEYWORDS: light-emitting nanofibers, random lasers, nanolasers, titania nanoparticles, mode-correlation, waveguides, electrospinning

$\mathrm{D}$ isordered materials with optical gain are the building blocks of random lasers, whose operation is based on the scattering properties of light. ${ }^{1}$ These devices can be useful for a wide range of applications, which include diagnostic ${ }^{2}$ and speckle-free $^{3}$ imaging, spectroscopic tools for monitoring biomaterials and structural deformations, ${ }^{4}$ and new laser projector schemes and optical tomography. ${ }^{5}$ A number of experimental $^{6-9}$ and theoretical ${ }^{10-13}$ approaches have been developed to rationalize the behavior and to tailor the performances of random lasers. In this framework, two main classes of devices are distinguished and experimentally observed. In so-called intensity (or incoherent) feedback random lasing (IFRL), the propagation of light can be described as a diffusional process with amplification, neglecting interference effects. ${ }^{10,14}$ This mechanism leads to smooth and narrow emission peaks, with full width at half-maximum (fwhm) of a few nm. Instead, in resonant feedback random lasing (RFRL), interference upon multiple scattering plays a major role, which might lead to some degree of spatial localization of the light modes, and to very narrow (fwhm down to sub-nm) lasing peaks, as found since $1999^{6}$ in numerous systems at the solid state. The RFRL to IFRL transition has been recently investigated by reducing the directionality of pumping photons. ${ }^{9,15}$ In this way, this transition could be associated with the coupling of modes which determines a condensation-like process, namely the onset of collective oscillations from $\mu \mathrm{m}$-sized titania clusters in a rhodamine solution. Extending the study of this transition to solid-state devices would be highly important to understand how the emission features and mode interactions of random lasers can be tailored. Indeed, regimes with different feedback or intermode coupling should be obtained not only by shaping the excitation beam as in previous reports, but also by varying the degree of disorder, through the composition and morphology of materials showing gain properties. ${ }^{16}$ These include a large variety of systems such as conjugated polymers, ${ }^{8}$ dyes in plastic matrices, subwavelength particles with plasmonenhanced scattering, ${ }^{17}$ cellulose fibers doped with $\mathrm{Au}$ nanoparticles, ${ }^{18}$ and light-emitting nanocomposites, ${ }^{19}$ usable in displays, organic lasers, and in nanophotonics.

Received: November 5, 2017

Published: December 18, 2017 

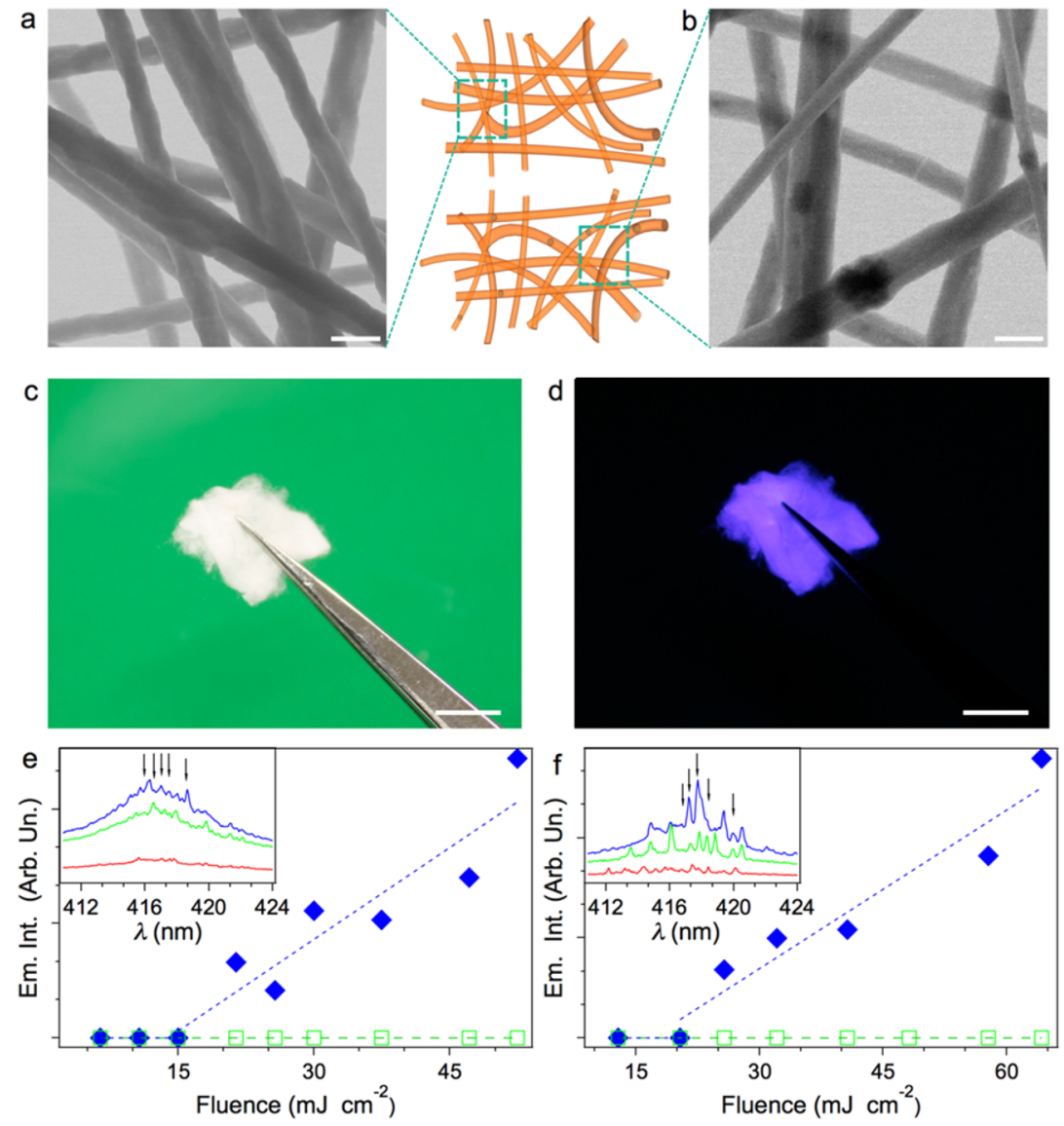

Figure 1. Morphology and emission characteristics of Fl-Cz-Fl/PS fiber random lasers. (a, b) Scanning transmission electron micrographs of Fl-Cz$\mathrm{Fl} / \mathrm{PS}$ fibers without (a) or with (b) $\mathrm{TiO}_{2}$ particles. Scale bar: $5 \mu \mathrm{m}$. Schemes of the obtained structures are also shown. (c, d) Mats of Fl-Cz-Fl/PS fibers under white (c) and UV illumination (d). Scale bar: $1 \mathrm{~cm}$. (e, f) Laser emission intensity vs excitation fluence for devices without (e) or with (f) $\mathrm{TiO}_{2}$ (full diamonds). Empty squares: PL intensity. Insets: emission spectra for increasing fluences (from bottom to top, (e) 26.7, 46.0, and 53.5 $\mathrm{mJ} \mathrm{cm}{ }^{-2}$ and (f) $24.6,44.9$, and $64.2 \mathrm{~mJ} \mathrm{~cm}^{-2}$ ). Vertical arrows in the insets point peaks at the wavelengths considered for correlation analysis.

Here we show how spectral characteristics and mode intensity correlation features can be tailored in random lasers made of electrospun polymer fibers with optical gain upon controlling local disorder by doping with $\mathrm{TiO}_{2}$ nanoparticles. Fibers serve as multifunctional component in the devices, providing stimulated emission, waveguiding along their longitudinal axis (multidirectional in disordered mats), and moderate scattering due to their refractive index $(\sim 1.6)$ and cylindrical body. ${ }^{20}$ These properties induce IFRL with highly interacting resonances, resulting in a strongly correlated random lasing emission. Clustered $\mathrm{TiO}_{2}$ particles provide additional elastic scattering and feedback in the complex material, and are found to drive the system to RFRL with discrete and more weakly interacting lasing modes. The onset of the RFRL-to-IFRL transition is also probed reducing guided radiation, through directionality selection for collected modes. These results are the first experimental evidence of the role of waveguiding in enhancing mode coupling in random lasers.

\section{RESULTS AND DISCUSSION}

We fabricate random lasers made of disordered mats of electrospun polystyrene (PS) fibers (Figure 1a) doped with the recently proposed 2,7-bis(9,9-diethylfluoren-2-yl)-9-(2ethylhexyl)carbazole (Fl-Cz-Fl) as gain material ${ }^{21}$ and with a further degree of disorder provided by optionally embedded $\mathrm{TiO}_{2}$ particles (Figure 1b). PS is chosen as thermoplastic polymer with refractive index about 1.6 and good optical transparency in the visible and near-infrared, leading to waveguides with low propagation losses and improved mode confinement compared to other transparent polymers. ${ }^{22}$ In addition, the elastic modulus (of the order of $1 \mathrm{GPa}$ ) and the thermal properties (glass-transition temperature, $T_{\mathrm{g}} \sim 100{ }^{\circ} \mathrm{C}$ ) of electrospun PS fibers ${ }^{23}$ make plastic lasers based on them being stable while operating at room temperature. $\mathrm{Fl}-\mathrm{Cz}-\mathrm{Fl}$ is a blue-emitting compound featuring fluorene and carbazole moieties arranged into 2,7-substitution pattern, and shows high emission quantum yield $(\sim 0.8)$ and large radiative decay rate $\left(\sim 10^{9} \mathrm{~s}^{-1}\right) .{ }^{21}$ Additionally, the twisted molecular structure and bulky peripheral moieties of Fl-Cz-Fl lead to high $T_{\mathrm{g}}(68$ ${ }^{\circ} \mathrm{C}$ ) ensuring formation of stable amorphous phase. The electrospinning technique is very effective in producing almost one-dimensional polymer structures, including light-emitting nanofibers, ${ }^{24}$ given that the used electrified solution shows a sufficient amount of macromolecule entanglements. ${ }^{25,26}$ Hybrid fibers can be straightforwardly electrospun, incorporating nanoparticles in organic filaments to tailor optical properties through the embedded components. Also, the method is highly convenient to realize random lasers made of light-emitting fibers with optical gain and assembled in slabs. Indeed, the critical thickness for lasing from slabs is given by $t_{\mathrm{CR}}=\pi\left(l_{\mathrm{G}} l_{\mathrm{T}} /\right.$ $3)^{1 / 2}$, where $l_{\mathrm{G}}$ and $l_{\mathrm{T}}$ indicate the gain length and the transport mean free path of the diffused photons, respectively. ${ }^{10}$ For 



Figure 2. (a) Matrix of plots showing the correlation among pairs of emission intensities, $I\left(\lambda_{j}\right)$ and $I\left(\lambda_{k}\right)$, for each pair of lasing wavelengths $\left(\lambda_{j}, \lambda_{k}\right)$ shown in the insets of Figure 1e,f and measured for Fl-Cz-Fl $/$ PS fibers. $\lambda_{1}=415.9 \mathrm{~nm}, \lambda_{2}=416.6 \mathrm{~nm}, \lambda_{3}=417.0 \mathrm{~nm}, \lambda_{4}=417.5 \mathrm{~nm}, \lambda_{5}=418.6 \mathrm{~nm}$. Continuous lines are linear fits to the data. Numbers in the top-left angles in each plot are the correlation coefficients. Histograms representing distributions, normalized to probability density, of the intensity measured for each wavelength at varying excitation pulse are displayed along the matrix diagonal. Excitation fluence $=46 \mathrm{~mJ} \mathrm{~cm}^{-2}$. (b) Single-shot emission spectra evolution upon increasing the number of excitation pulses.

lasing dyes in plastic matrices, $l_{\mathrm{G}}$ might be of the order of a few hundreds of $\mu \mathrm{m}$, whereas $l_{\mathrm{T}}$ in our membranes of electrospun fibers is estimated in the range $2-10 \mu \mathrm{m}$ by coherent backscattering (CBS) experiments. This leads to $t_{\mathrm{CR}}$ values of about $100 \mu \mathrm{m}$, which are well within the range of thickness which is easily obtained by electrospinning. The here realized organic fibers show an average diameter of about $4 \mu \mathrm{m}$ (Figure S1a in the Supporting Information), with micron-size roughness and roughly cylindrical body as shown in Figure 1a. When inserted in the electrospun solution, $\mathrm{TiO}_{2}$ particles $(\sim 15-23$ $\mathrm{nm}$, Figure S1b) can conglomerate within fibers, forming clusters with size up to a few $\mu \mathrm{m}$ (Figure 1b), thus, introducing additional elastic scattering for transported light. Exemplary photographs of the electrospun mats under white illumination conditions and under UV excitation are shown in Figure 1c and $\mathrm{d}$, respectively. The different sample morphologies and compositions can so lead to varied emission characteristics, as shown in Figure 1e,f. Upon excitation with about 10 ns pulses at $355 \mathrm{~nm}$ and with a stripe excitation geometry (see Methods), lasing spectra from samples without $\mathrm{TiO}_{2}$ particles exhibit a single peak at about $417 \mathrm{~nm}$ with fwhm of about $6 \mathrm{~nm}$. The spectra are mostly smooth and only slightly featured at their top, and are ascribed to IFRL. Instead, emission spectra from fibers doped with $\mathrm{TiO}_{2}$ particles are spiky, with several narrow ( $\mathrm{fwhm} \cong 0.3 \mathrm{~nm}$ ) peaks whose wavelength is quite stable upon changing excitation fluence. These features are clearly to be ascribed to RFRL. Well-defined excitation thresholds are found for lasing emission, at about $15 \mathrm{~mJ} \mathrm{~cm}^{-2}$ and at about $20 \mathrm{~mJ}$ $\mathrm{cm}^{-2}$ for purely organic and for $\mathrm{TiO}_{2}$-doped fibers, respectively, while the background spontaneous emission, that is, photoluminescence (PL) signal grows weakly and largely linearly upon increasing the excitation fluence. One might also expect a significant contribution to mode excitation coming from indirect pumping by amplified spontaneous emission (ASE) from the surrounding regions, which is waveguided in the organic slab constituted by the polymer nonwoven. The persistence of ASE components directionally guided along networks of randomly oriented nanofibers has been shown in previous work. ${ }^{27}$ The increase of the lasing threshold upon embedment of the $\mathrm{TiO}_{2}$ particles is therefore related to the correspondingly decreased characteristic scattering mean free path, experienced by the directional pumping from ASE along the excitation stripe. ${ }^{28}$ Various methods would allow the excitation threshold of fiber-based random lasers to be decreased, including the exploitation of plasmon-enhanced light scattering by incorporated Au nanoparticles. ${ }^{18}$ Overall, the addition of $\mathrm{TiO}_{2}$ nanoparticles and the increased local degree of disorder clearly drive the fiber-based random laser from a multimode IFRL regime to the RFRL regime, featuring few discrete lasing modes. In this respect, this transition from a smoother to a more spiky emission is here demonstrated to be associated in a clear way with the compositional and morphological complexity of the disordered medium, offering 
a

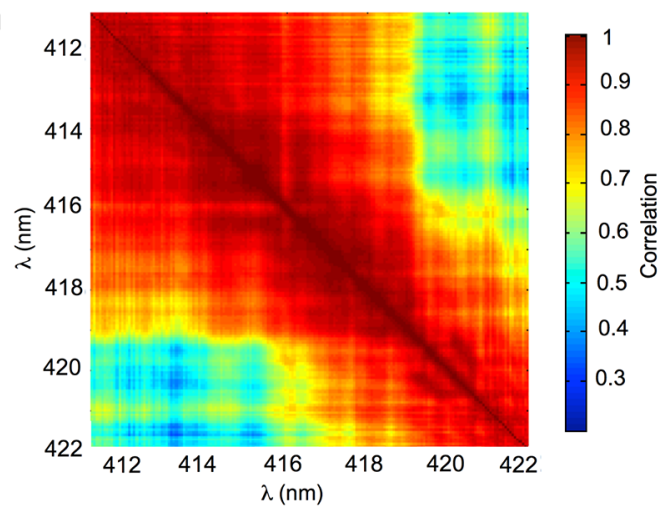

C

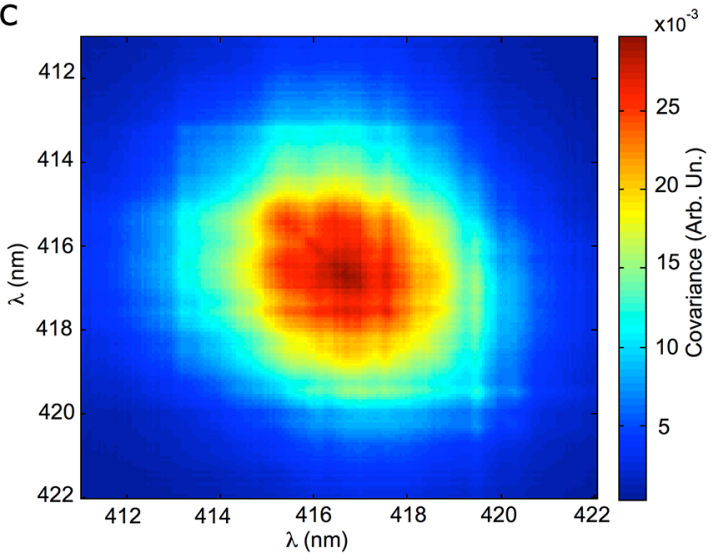

b



d

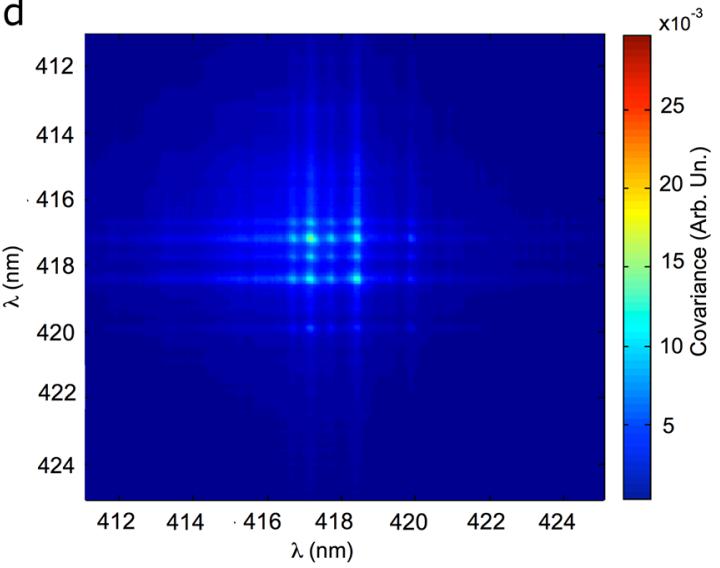

Figure 3. (a, b) Map showing the correlation function of $N$ single-shot emission spectra acquired from a sample without (a) or with $(\mathrm{b}) \mathrm{TiO}_{2}$ particles vs wavelength. $N=44-83$. Excitation fluences $=46.0 \mathrm{~mJ} \mathrm{~cm}{ }^{-2}(\mathrm{a}), 42.8 \mathrm{~mJ} \mathrm{~cm}^{-2}(\mathrm{~b})$. The dashed rectangle in (b) highlights a region with higher internal correlation and lower $C$ values at its vertexes, evidencing reduced intermode interactions in $\mathrm{TiO}_{2}$-doped fiber random lasers. (c, d) Corresponding covariance maps.

a direct method to tailor spectral characteristics by material engineering.

Two different scattering components are present in $\mathrm{TiO}_{2}-$ doped fiber mats, which are the polymer filaments with highly elongated shape $\mathrm{e}^{20}$ and with waveguiding capability along their axis, ${ }^{29}$ and the inorganic particles, respectively. To better rationalize the contribution of the different scatterers, we consider the correlation of intensities of different modes in the emission spectra from samples with and without $\mathrm{TiO}_{2}$ nanoparticles. The Pearson's correlation coefficient, $C$, for the intensity fluctuations at a couple of wavelengths $\left(\lambda_{j}, \lambda_{k}\right)$ is

$$
\begin{aligned}
& C\left(\lambda_{j}, \lambda_{k}\right)= \\
& \frac{\sum_{i=1}^{N}\left[I_{i}\left(\lambda_{j}\right)-\bar{I}\left(\lambda_{j}\right)\right]\left[I_{i}\left(\lambda_{k}\right)-\bar{I}\left(\lambda_{k}\right)\right]}{\sqrt{\sum_{i=1}^{N}\left[I_{i}\left(\lambda_{j}\right)-\bar{I}\left(\lambda_{j}\right)\right]^{2}} \sqrt{\sum_{i=1}^{N}\left[I_{i}\left(\lambda_{k}\right)-\bar{I}\left(\lambda_{k}\right)\right]^{2}}}
\end{aligned}
$$

where $\bar{I}$ indicates the average over the ensemble given by $N$ analyzed shots (excitation pulses). The $C$ coefficient is zero for completely uncorrelated peak intensities, and reaches a unity maximum value when peaks are fully correlated. The intermode spectral correlation is here studied for $j, k=1, \ldots, 5$, namely, for five relevant wavelengths highlighted by arrows in the insets of Figure 1e,f. The emission from our interconnected network of fibers under directional excitation generally exhibits correlation coefficients (0.76-0.97), which are significantly larger than those reported for uncorrelated, RFRL modes in solutions with scattering nanoparticles. ${ }^{9}$ The role of waveguiding filaments in promoting mode intensity correlation is also supported by the higher average correlation found for Fl-Cz-Fl/PS fibers (0.94 \pm $0.02)$ compared to mats with scattering particles $(0.89 \pm 0.05)$. These results are shown in the correlation matrix plots in Figure 2a and in Figure S2a, respectively. Here the normalized histograms showing the frequencies of the measured intensities at fixed wavelengths (from shot to shot) are plotted along the matrix diagonal, whereas the off-diagonal panels in Figure 2a display intensity data interdependencies of selected couples of modes, evidencing high correlation in each pair of intensities, with $C$ values close to unity. Instead, scattering particles lead to a decorrelation of the overall emission spectra (Figure S2) and to an increase of the spread of the intensity data around the diagonal line (Figures S3 and S4). This is also reflected in the relatively higher standard deviations of the correlations found for samples with nanoparticles, which indicates that $C$ significantly depends on the pairs of wavelengths chosen, namely, on the specific modes involved. In Figure 2b, we show the shot-to-shot evolution of emission spectra for $\mathrm{Fl}-\mathrm{Cz}-\mathrm{Fl} / \mathrm{PS}$ fibers. Established modes have quite stable intensity at different excitation shots, whereas for $\mathrm{TiO}_{2}$-doped fibers one finds larger fluctuations over time, suggesting a stochastic dependence in the onset of resonances (Figure S2b).

To better highlight how $C$ depends on wavelengths, we plot two-dimensional correlation maps for the spectra of $\mathrm{Fl}-\mathrm{Cz}-\mathrm{Fl} /$ PS fibers without and with $\mathrm{TiO}_{2}$ in Figure $3 \mathrm{a}$ and b, respectively. The highly correlated spectral region of the fiber random laser is clearly appreciable around the autocorrelation diagonal (top-left to bottom-right map angles in Figure 3a). Upon embedding $\mathrm{TiO}_{2}$ nanoparticles into the mat of light- 
emitting fibers, the map becomes textured in a very different way, featuring a grid pattern superimposed to the autocorrelation diagonal (Figure 3b). This highlights lower intercorrelation of the main peak wavelengths, suggestive of relatively poor interaction established between spectrally adjacent modes. These findings are in agreement with recent results reported for the random lasing fluctuations in gelatinous gain media with colloidal scatterers. ${ }^{30}$ Indeed, points defined by crossing vertical and horizontal lines are highly correlated only if belonging to the autocorrelation diagonal (red dots in the map in Figure $3 b$ ), whereas adding particles in the laser materials leads to the emergence of discrete pairs of lasing peaks with correlations values $<0.5$ (cyan, off-diagonal dots in Figure $3 \mathrm{~b}$ ), namely, with modest intensity correlation. ${ }^{31}$ Squared regions with vertexes in such dots exhibit high correlations within their area and a lower one on their sides, as exemplified by the rectangle displayed in the map.

The correlation and spectral features can be also conveniently analyzed by covariance mapping. ${ }^{32}$ To this aim we plot the covariance $\left.\left(\sum_{i=1}^{N}\left[I_{i}\left(\lambda_{j}\right)-\bar{I}\left(\lambda_{j}\right)\right]\left[I_{i}\left(\lambda_{k}\right)-\bar{I}\left(\lambda_{k}\right)\right]\right) / N\right)$ for emission intensities at each pair of wavelengths (Figure $3 c, d$ ), which directly indicates how the two variables $I\left(\lambda_{j}\right)$ and $I\left(\lambda_{k}\right)$ change together from shot to shot. For Fl-Cz-Fl/PS fibers, the resulting map has a symmetric shape (Figure 3c), where concentric ellipses of different colors correspond to different levels of emission intensity (i.e., the decrease of intensity at the tails of the lasing peak occurs in a similar way for any shot). Instead, the grid pattern found for the $C$-map is even more clear in the covariance map for lasing fibers doped with $\mathrm{TiO}_{2}$ (Figure 3d).

For random lasers made of gain solutions with nanoparticle scatterers, excited with spots of different shapes, a mode-locking transition has been associated with the change from resonant to incoherent feedback regimes, and with the directionality of excitation and the spatial overlap of modes. ${ }^{9}$ Spatially distant or weakly overlapping modes might interact only partly, which would results in low correlation. Here, with identical excitation geometry $\mathrm{Fl}-\mathrm{Cz}-\mathrm{Fl} / \mathrm{PS}$ fibers exhibit either IFRL or RFRL spectral characteristics, depending on $\mathrm{TiO}_{2}$ doping, while the intensity correlation is high on average for both the investigated samples, evidencing mode interaction taking a role even in the RFRL regime. This peculiar behavior is due to various interplaying mechanisms which affect photon transport in the complex material composed by nanofibers. On one side, electrospun light-emitting polymer filaments might show, in addition to light-scattering whose form factor depends on the lasing wavelength and on the fiber cross-section, ${ }^{20}$ significant waveguiding properties. In our setup the excitation stripe is large enough to pump a large number of fibers (Figure 4a), thus, triggering different waveguided modes along various directions. The loss coefficient, $\gamma$, for self-emitted light propagating along the length of individual electrospun fibers are down to less than $100 \mathrm{~cm}^{-1}$, corresponding to effective photon transport over many tens of $\mu \mathrm{m},{ }^{29}$ which could favor coupling of otherwise poorly overlapping modes thus leading to correlated lasing modes. Figure $4 \mathrm{~b}$ shows an exemplary fluorescence microscopy image showing an excited Fl-Cz-Fl/ PS fiber with propagating light exiting the two tips of the polymer filament, which are distant about $120 \mu \mathrm{m}$. On the contrary, $\mathrm{TiO}_{2}$ particles disfavor photon transport along the organic material, possibly developing resonant cavities in the slab as a route to modal selection and consequently to very a



b
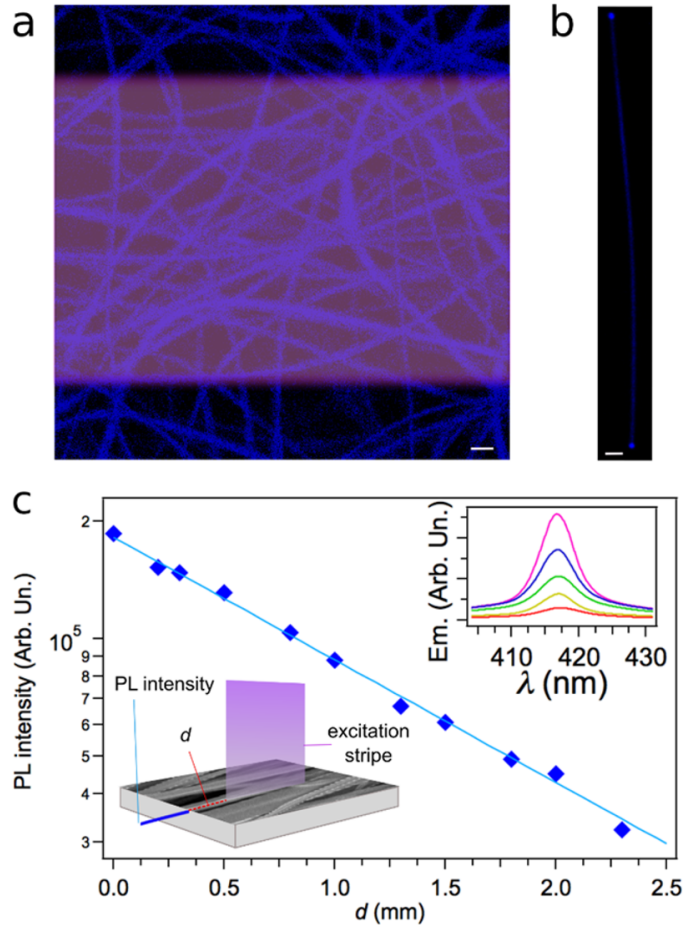

Figure 4. Waveguiding properties of Fl-Cz-Fl/PS fibers. (a) Confocal fluorescence micrograph of a mat of random fibers with the superposition of the excitation stripe (violet region, in scale). Scale bar: $5 \mu \mathrm{m}$. (b) Fluorescence image of an individual fiber with bright tips highlighting waveguiding of self-emitted light. The sample is excited by a UV lamp. Scale bar: $5 \mu \mathrm{m}$. (c) Spatial decay of emitted light intensity (diamonds) guided along $\mathrm{Fl}-\mathrm{Cz}-\mathrm{Fl} / \mathrm{PS}$ fibers, deposited on a quartz substrate, as a function of distance, $d$, from photoexcitation. The continuous line is the best fit of the experimental data by an exponential decay (see text). Bottom inset: scheme of the experimental configuration for waveguiding characterization. Top inset: collected spectra for (from top to bottom) $d=0,0.3,0.8,1.8$, and $2.5 \mathrm{~mm}$.

narrow lasing peaks. The intensity correlation of optical modes is correspondingly weakened. Through the formation of random ring resonators, a similar behavior has been found in dye-doped fibers with deterministic, comb-like spectral emission. ${ }^{33}$ Unlike previous works, however, our system is not composed by well-established cavities generated during fiber deposition, featuring instead a complex network of organic or hybrid filaments with significant diffusive behavior for transported light. Here the waveguiding effect is quantified by measuring the emission intensity as a function of the varying distance, $d$, of the excitation region from the emitting edge of aligned $\mathrm{Fl}-\mathrm{Cz}-\mathrm{Fl} / \mathrm{PS}$ fibers as schematized in the bottom inset of Figure $4 \mathrm{c}$. The decrease of the output intensity upon increasing $d$ (top inset of Figure 4c) is then fitted by a law, $I_{\mathrm{PL}}=$ $I_{0} e^{-\gamma d}$, where $I_{0}$ is a constant, providing a value of the loss coefficient as low as $7.2 \mathrm{~cm}^{-1}$ (Figure 4c). This value is comparable to propagation loss coefficients of active polymer slab waveguides, ${ }^{34,35}$ that is indicative of effective light transport along the disordered network of fibers. Waveguiding over millimeter distances is likely favored by both the optical confinement properties of individual fibers and by interfiber coupling. These properties of electrospun fibrous materials makes them highly interesting for random lasing applications, as well as for investigating coupling regimes promoted by enhanced light transport. 
We design specific experiments to test further how waveguiding properties of the electrospun fibers affect intermode correlation. To this aim, photon transport along the fiber length is intentionally suppressed by embedding the mats (without $\mathrm{TiO}_{2}$ doping) in a resin matrix, thus reducing the refractive index contrast $(\Delta n)$ between the internal and the external regions of the cylindrical, dielectric waveguides. Leading to higher optical leakages from fibers, this is found to induce a relevant change in the covariance for emission intensities from different wavelengths. The resulting map is shown in Figure 5a. Correspondingly, we find $C$ values close to


Figure 5. Impact of waveguiding on intermode intensity correlation. (a) Covariance map for Fl-Cz-Fl/PS fibers embedded in resin. Excitation fluence: $10.7 \mathrm{~mJ} \mathrm{~cm}{ }^{-2}$. Exemplary spectra from the resinincorporated random lasers are shown in Figure S5a. (b) Covariance map for Fl-Cz-Fl/PS fibers deposited on quartz, for emission collected with a sample holder-detection optical fiber at an angle of $45^{\circ}$ (scheme in Figure S5b). Excitation fluence: $46.0 \mathrm{~mJ} \mathrm{~cm}{ }^{-2}$.

unity which are signature of enhanced IFRL character. Furthermore, we calculate the covariance map for light emitted by random $\mathrm{Fl}-\mathrm{Cz}-\mathrm{Fl} / \mathrm{PS}$ fibers (without $\mathrm{TiO}_{2}$ particles and without resin), upon positioning the mat at an angle of $45^{\circ}$ $\left( \pm 2^{\circ}\right)$ with respect to the collecting optical fiber (Figure S5a). This method allows for better decoupling waveguided radiation, propagating in fibers, and detected light, the latter thus being relatively enriched in the scattered component. Again, the resulting map starts to resembling a grid pattern, namely it highlights relatively decorrelated emission peaks similarly to RFRL devices incorporating scattering particles (Figure 5b). This is also supported by a decrease of the correlation coefficient to $0.82 \pm 0.09$. Similar considerations, showing a transition toward spikiness, hold for emission spectra (Figure S5b).

Previous studies have shown that in dye solutions with titania particles, spiky lasing spectra are largely restricted to weakly scattering systems, while multiple light scattering events or lower gain more likely lead to smooth lasing spectra as in IFRL. ${ }^{36}$ This is interpreted in terms of the diffusive and omnidirectional character of random lasers with relatively stronger scattering, where many modes share the same gain medium. As we evidence by changing the photon collection direction (Figure $5 \mathrm{~b}$ ), in random lasers with fibers, relevant elements, including waveguiding, are present instead, which introduce directionality, namely, angular mode selectivity in the emission. On the other hand, multidirectional waveguides constituted by the electrospun fibers are likely to provide the material with enhanced modal correlation. Indeed, at variance with early works exploiting titanium dioxide powders in solutions, ${ }^{9,37}$ here highly correlated spectral modes are found even by a stripe excitation geometry. Since such geometry is likely to lead to a lower intrinsic interaction of distant modes, locking different emissive regions through the network of nanofibers might be critically important to establish correlation in different and narrow peaks observed in this work. In this way, also RFRL peaks, in principle originating from spatially decoupled positions, and not only IFRL regions, can undergo collective oscillations. In this respect, the decrease of optical losses provided by light-emitting nanofibers ${ }^{29}$ can be therefore relevant to prevent the inhibition of the coupling dynamics ${ }^{15}$ and to favor a mode correlation which is significant also in RFRL regime. In addition, increasing the scattering strength by adding nanoparticles may lead to a double effect including directionality suppression (which would strengthen the IFRL character of the emission) as well as development of extra resonant cavities. These would interact through the network of waveguiding polymer filaments, leading the device to RFRL with significant though relatively weaker intensity correlation. Our results suggest that this latter mechanism, not present in experiments in solution, ${ }^{9,37}$ might prevail in solid-state nanomaterials.

\section{CONCLUSIONS}

Overall, these findings show how tunable, multicomponent disordered morphologies might allow the intensity correlation of light modes to be tailored, activated or suppressed in hybrid nano- and microstructures. Controlling the transition from IFRL to RFRL character in random lasing materials represents an important step toward engineering light confinement and emission in these systems. The occurrence of a regime of mode-locked lasing with intensity-correlated modes was envisaged at low degrees of disorder, whereas increasing disorder should drive the lasing system toward modes coherent in phase but not in intensity. ${ }^{16}$ Our work is a first experimental study focusing on mode-intensity correlation in structurally disordered, fibrous materials. In this framework electrospun fibers with controlled nanoparticle doping are a versatile platform, which allows morphology and composition of hybrid lasers to be varied in a controlled way, materials with effective waveguiding networks to be realized, and resonances to be tailored, which can establish new design rules for amorphous photonics. 


\section{METHODS}

Light-Emitting Nanofibers. Polymer fibers are electrospun from a solution of $300 \mathrm{mg} / \mathrm{mL}$ of PS (Sigma-Aldrich, molecular weight $=192000 \mathrm{Da})$ in chloroform (Sigma-Aldrich), dissolving $\mathrm{Fl}-\mathrm{Cz}-\mathrm{Fl}$ at $5 \%(\mathrm{w} / \mathrm{w})$ with respect to PS. The synthesis procedure of $\mathrm{Fl}-\mathrm{Cz}-\mathrm{Fl}$ has been reported elsewhere. ${ }^{21}$ In parallel, another solution is prepared adding $\mathrm{TiO}_{2}$ nanoparticles (titanium(IV) oxide nanopowder, Sigma Aldrich), at a concentration of $1 \%(\mathrm{w} / \mathrm{w})$ with respect to PS. The Norland Optical Adhesive 68 (NOA68) is used to optionally embed electrospun fibers. Solutions are stirred for $12 \mathrm{~h}$, loaded into a 1 $\mathrm{mL}$ syringe, delivered at a constant flow rate of $1 \mathrm{~mL} / \mathrm{h}$ through a metal needle (21 gauge) by a peristaltic pump (33 Dual Syringe Pump, Harvard Apparatus Inc., Holliston, MA) and electrospun at ambient conditions. The needle is connected to a high-voltage power supply (EL60R0.6-22, Glassman High Voltage, High Bridge, NJ), applying a bias of $13 \mathrm{kV}$. Fibers are collected on a grounded metallic plate at a distance of $10 \mathrm{~cm}$ from the needle. Arrays of uniaxially aligned $\mathrm{Fl}-\mathrm{Cz}-\mathrm{Fl} / \mathrm{PS}$ fibers are also fabricated by using a rotating collector $(4000 \mathrm{rpm})$. For encapsulation, the fibrous mats are embedded NOA68, which is then cured by exposure to UV light $\left(3 \mathrm{~mW} / \mathrm{cm}^{2}\right)$ for $3 \mathrm{~min}$. A sample consisting of a NOA68 layer sandwiched between two glass substrates and without fibers is prepared to measure the resin transmission (Figure S5b).

Morphological and Optical Characterization. The morphology of electrospun fibers is investigated by scanning transmission electron microscopy (Nova NanoSEM 450, FEI) with an acceleration voltage of $30 \mathrm{kV}$. Fluorescence micrographs are collected by an inverted microscope (Nikon) working in epi-layer configuration. The fibers are excited by a mercury lamp, while the emission is collected by the same objective and imaged by a charge coupled device (CCD) camera. Confocal fluorescence micrographs are obtained by exciting the sample with a diode laser $(\lambda=405 \mathrm{~nm})$, focused through an objective lens $(20 \times$, numerical aperture $=0.5)$. The emission from the fibers is then collected by the same objective and analyzed by a multianode photomultiplier. A spectrophotometer (PerkinElmer, Lambda 950) is used for transmittance measurements. The light mean free path of nanofibers mats is measured through CBS by a He:Ne laser beam with wavelength at $633 \mathrm{~nm}$. The backscattered light intensity angular profile is measured by a CCD Camera (iDUS, Andor).

Random Lasers. Random lasing is achieved by pumping samples with the third harmonic $(\lambda=355 \mathrm{~nm})$ of a Nd:YAG laser beam (repetition rate $=10 \mathrm{~Hz}$, pulse duration $\cong 10 \mathrm{~ns}$ ), focused in a rectangular stripe $(55 \mu \mathrm{m} \times 1.9 \mathrm{~mm})$. The emission is then collected from the sample edge, coupled to an optical fiber and analyzed by a monochromator (iHR320, Jobin Yvon) equipped with a CCD detector (Symphony, Jobin Yvon). Random lasing thresholds are measured by systematically varying the excitation fluence, and series of single-shot spectra are acquired with an integration time of $50 \mathrm{~ms}$. By keeping fixed the pump fluence, optical losses are measured through the emitted light intensity by moving the excitation stripe away from the sample emitting edge.

\section{ASSOCIATED CONTENT}

\section{S Supporting Information}

The Supporting Information is available free of charge on the ACS Publications website at DOI: 10.1021/acsphotonics.7b01329.
Additional details on nanofiber morphology, mode intensity correlation measurements on devices embedding nanoparticles, and spectroscopy experiments (PDF).

\section{AUTHOR INFORMATION}

\section{Corresponding Authors}

*E-mail: andrea.camposeo@nano.cnr.it.

*E-mail: dario.pisignano@unipi.it.

ORCID 우

Karolis Kazlauskas: 0000-0001-7900-0465

Dario Pisignano: 0000-0003-3758-5199

Notes

The authors declare no competing financial interest.

\section{ACKNOWLEDGMENTS}

The research leading to these results has received funding from the European Research Council under the European Union's Seventh Framework Programme (FP/2007-2013)/ERC Grant Agreement No. 306357 (ERC Starting Grant “NANO-JETS”). The Apulia Networks of Public Research Laboratories WAFITECH (09) and MITT (13), and the Fund for Development and Cohesion 2007-2013 APQ Apulia Region Research "Regional program for smart specialization and social and environmental sustainaibility" (YP9DNY7) are also acknowledged. K.K. and S.J. acknowledge funding by a grant (No. LJB-3/2015) from the Research Council of Lithuania. V. Fasano is acknowledged for CBS measurements. A. Fornieri is acknowledged for scanning electron microscopy on nanoparticles.

\section{REFERENCES}

(1) Wiersma, D. S. The physics and applications of random lasers. Nat. Phys. 2008, 4, 359-367.

(2) Polson, R. C.; Vardeny, Z. V. Random lasing in human tissues. Appl. Phys. Lett. 2004, 85, 1289-1291.

(3) Redding, B.; Choma, M. A.; Cao, H. Speckle-free laser imaging using random laser illumination. Nat. Photonics 2012, 6, 355-359.

(4) Song, Q.; Xiao, S.; Xu, Z.; Liu, J.; Sun, X.; Drachev, V.; Shalaev, V. M.; Akkus, O.; Kim, Y. L. Random lasing in bone tissue. Opt. Lett. 2010, 35, 1425-1427.

(5) Graydon, O.; Cao, H. Random thoughts. Nat. Photonics 2013, 7, 164-165.

(6) Cao, H.; Zhao, Y. G.; Ho, S. T.; Seelig, E. W.; Wang, Q. H.; Chang, R. P. Random laser action in semiconductor powder. Phys. Rev. Lett. 1999, 82, 2278-2281.

(7) Fallert, J.; Dietz, R. J.; Sartor, J.; Schneider, D.; Klingshirn, C.; Kalt, H. Co-existence of strongly and weakly localized random laser modes. Nat. Photonics 2009, 3, 279-282.

(8) Tulek, A.; Polson, R. C.; Vardeny, Z. V. Naturally occurring resonators in random lasing of $\pi$-conjugated polymer films. Nat. Phys. 2010, 6, 303-310.

(9) Leonetti, M.; Conti, C.; Lopez, C. The mode-locking transition of random lasers. Nat. Photonics 2011, 5, 615-617.

(10) Wiersma, D. S.; Lagendijk, A. Light diffusion with gain and random lasers. Phys. Rev. E: Stat. Phys., Plasmas, Fluids, Relat. Interdiscip. Top. 1996, 54, 4256-4265.

(11) Apalkov, V. M.; Raikh, M. E.; Shapiro, B. Random resonators and prelocalized modes in disordered dielectric films. Phys. Rev. Lett. 2002, 89, 016802.

(12) Türeci, H. E.; Ge, L.; Rotter, S.; Stone, A. D. Strong interactions in multimode random lasers. Science 2008, 320, 643-646.

(13) Conti, C.; Leonetti, M.; Fratalocchi, A.; Angelani, L.; Ruocco, G. Condensation in disordered lasers: Theory, $3 \mathrm{D}+1$ simulations, and experiments. Phys. Rev. Lett. 2008, 101, 143901. 
(14) Letokhov, V. S. Generation of light by a scattering medium with negative resonance absorption. Sov. Phys. Jetp. 1968, 26, 835-840.

(15) Leonetti, M.; Conti, C.; López, C. Dynamics of phase-locking random lasers. Phys. Rev. A: At., Mol., Opt. Phys. 2013, 88, 043834.

(16) Antenucci, F.; Conti, C.; Crisanti, A.; Leuzzi, L. General phase diagram of multimodal ordered and disordered lasers in closed and open cavities. Phys. Rev. Lett. 2015, 114, 043901.

(17) Dice, G. D.; Mujumdar, S.; Elezzabi, A. Y. Plasmonically enhanced diffusive and subdiffusive metal nanoparticle-dye random laser. Appl. Phys. Lett. 2005, 86, 131105.

(18) Zhang, R.; Knitter, S.; Liew, S. F.; Omenetto, F. G.; Reinhard, B. M.; Cao, H.; Dal Negro, L. Plasmon-enhanced random lasing in biocompatible networks of cellulose nanofibers. Appl. Phys. Lett. 2016, 108, 011103.

(19) Sanchez, C.; Lebeau, B.; Chaput, F.; Boilot, J. P. Optical properties of functional hybrid organic-inorganic nanocomposites. Adv. Mater. 2003, 15, 1969-1994.

(20) Persano, L.; Moffa, M.; Fasano, V.; Montinaro, M.; Morello, G.; Resta, V.; Spadaro, D.; Gucciardi, P. G.; Maragò, O. M.; Camposeo, A.; Pisignano, D. Optimization of electrospinning techniques for the realization of nanofiber plastic lasers. Proc. SPIE 2016, 9745, 97450R. (21) Baronas, P.; Kazlauskas, K.; Kreiza, G.; Jankauskas, V.; Tomkeviciene, A.; Simokaitiene, J.; Grigalevicius, S.; Grazulevicius, J. V.; Jursenas, S. Differently linked fluorene-carbazole triads for light amplification. Dyes Pigm. 2015, 123, 370-379.

(22) Ramírez, M. G.; Morales-Vidal, M.; Navarro-Fuster, V.; Boj, P. G.; Quintana, J. A.; Villalvilla, J. M.; Retolaza, A.; Merino, S.; DíazGarcía, M. A. Improved performance of perylenediimide-based lasers. J. Mater. Chem. C 2013, 1, 1182-1191.

(23) Pai, C.-L.; Boyce, M. C.; Rutledge, G. C. Morphology of porous and wrinkled fibers of polystyrene electrospun from dimethylformamide. Macromolecules 2009, 42, 2102-2114.

(24) Persano, L.; Camposeo, A.; Pisignano, D. Active polymer nanofibers for photonics, electronics, energy generation and micromechanics. Prog. Polym. Sci. 2015, 43, 48-95.

(25) Reneker, D. H.; Chun, I. Nanometre diameter fibres of polymer, produced by electrospinning. Nanotechnology 1996, 7, 216-223.

(26) Li, D.; Xia, Y. Electrospinning of nanofibers: reinventing the wheel? Adv. Mater. 2004, 16, 1151-1170.

(27) Morello, G.; Moffa, M.; Girardo, S.; Camposeo, A.; Pisignano, D. Optical Gain in the Near Infrared by Light-Emitting Electrospun Fibers. Adv. Funct. Mater. 2014, 24, 5225-5231.

(28) Leonetti, M.; Lopez, C. Random lasing in structures with multiscale transport properties. Appl. Phys. Lett. 2012, 101, 251120.

(29) Fasano, V.; Polini, A.; Morello, G.; Moffa, M.; Camposeo, A.; Pisignano, D. Bright light emission and waveguiding in conjugated polymer nanofibers electrospun from organic salt added solutions. Macromolecules 2013, 46, 5935-5942.

(30) Merrill, J. W.; Cao, H.; Dufresne, E. R. Fluctuations and correlations of emission from random lasers. Phys. Rev. A: At., Mol., Opt. Phys. 2016, 93, 021801.

(31) Taylor, R. Interpretation of the correlation coefficient: a basic review. J. Diagn. Med. Sonography 1990, 6, 35-39.

(32) Frasinski, L. J.; Codling, K.; Hatherly, P. A. Covariance Mapping: A Correlation Method Applied to Multiphoton Multiple Ionization. Science 1989, 246, 1029-1031.

(33) Krämmer, S.; Vannahme, C.; Smith, C. L.; Grossmann, T.; Jenne, M.; Schierle, S.; Jørgensen, L.; Chronakis, I. S.; Kristensen, A.; Kalt, H. Random-Cavity Lasing from Electrospun Polymer Fiber Networks. Adv. Mater. 2014, 26, 8096-8100.

(34) McGehee, M. D.; Gupta, R.; Veenstra, S.; Miller, E. K.; DiazGarcia, M. A.; Heeger, A. J. Amplified spontaneous emission from photopumped films of a conjugated polymer. Phys. Rev. B: Condens. Matter Mater. Phys. 1998, 58, 7035-7039.

(35) Xia, R.; Heliotis, G.; Bradley, D. D. C. Fluorene-based polymer gain media for solid-state laser emission across the full visible spectrum. Appl. Phys. Lett. 2003, 82, 3599-3601.

(36) El-Dardiry, R. G.; Mooiweer, R.; Lagendijk, A. Experimental phase diagram for random laser spectra. New J. Phys. 2012, 14, 113031.
(37) Leonetti, M.; Conti, C.; Lopez, C. Non-locality and collective emission in disordered lasing resonators. Light: Sci. Appl. 2013, 2, e88. 